\title{
Towards Dynamic Virtual Enterprises
}

\author{
Vaggelis Ouzounis, Volker Tschammer \\ ECCO - Electronic Commerce Center of Competence, GMD-Fokus, Kaiserin-Augusta-Allee 31, \\ D-10589, Berlin, Germany, Email: (ouzounis, tschammer)@fokus.gmd.de
}

\begin{abstract}
Virtual Enterprises (VEs) enable the deployment of distributed business processes among different partners in order to shorten development and manufacturing cycles, reduce time to market and operational costs, increase customer satisfaction, and operate on global scale and reach. Dynamic virtual enterprises are an emerging category of VEs, where the different partners are being selected dynamically during business process execution based on market-driven criteria and negotiation. In this paper, we discuss concepts and technologies that are considered to satisfy key requirements of dynamic virtual enterprises, and propose DIVE, a framework for the specification, execution and management of shared business processes in dynamic virtual enterprises.
\end{abstract}

\section{INTRODUCTION}

In the digital economy, companies are continuously seeking for new ways of business in order to cope with the increasing competitive pressure generated by the global market place. The need for global scale and reach, short development and manufacturing cycles, reduced time-to-market and operational costs, increased customer satisfaction, and rapid adaptation to new market changes has led companies to intensify automation, collaboration, and distribution (Applegate 96, Malone 91, Ouzounis 98a). The Internet is increasingly becoming the general basis for such purposes together with its advanced services, including electronic market places and the World Wide Web. New organisational forms develop, like collaborative commerce and virtual enterprises. In such virtual, collaborative organisations, partners, particularly small and medium enterprises (SMEs), can concentrate on their own core-competence and exploit partners' resources and capacities. 
In this paper, we discuss concepts and technologies that satisfy key requirements of dynamic virtual enterprises, and propose DIVE, a framework for the specification, execution, and management of shared business processes.

\section{DYNAMIC VIRTUAL ENTERPRISES}

Distribution of tasks and co-operation has been a central aspect of trade and business since centuries. Manifold relationships between enterprises and parts of enterprises, often invisible for the customer, have contributed to the goal of satisfying the customer's needs for timely and high quality services. In the digital economy, where many of those relationships are realised via the Internet, new forms of business must develop which support communication and co-operation between distributed and automated business processes. Such Internet-based, collaborative organisations are usually called virtual organisations or virtual enterprises.

Virtual enterprises are characterised by the following properties (Ouzounis 98b, Ouzounis 99):

- the processes which take share in the business are distributed via distant partner organisations,

- the partners are autonomous, i.e. there need not be other co-operation or dependency outside their engagement in the virtual enterprise,

- the partners engaged in the virtual enterprise are organised internally in a way that they can delegate some or all of their business processes and resources totally or partially to the virtual organisation,

- the partners have made agreements about common goals, negotiated how to reach these goals, assigned roles and distributed responsibilities, defined how the processes and resources have to be introduced, shared, used, and administered, developed procedures of problem handling, and made agreements about how to share profits and losses,

- the partners use common information and communication infrastructures in order to support collaboration and co-ordination during the life-cycle of the virtual enterprise.

Virtual enterprises are not a new concept in management studies (Malone 91, Ouzounis 99, Camarinha-Matos 99). Some of the big manufacturing companies have already business relationships with their suppliers and customers based on electronic procedures and protocols, such as EDI (Filos 00). These "virtual" business relationships enable the sharing of business processes and resources via electronic information and communication services. However, the level of integration and the usage characteristics of information and communication technologies vary and are often not optimal. Most of the activities are still performed manually and the cost of implementing and integrating electronic solutions as well as the time required to deploy virtual enterprise concepts are high (Lin 96). 
Based on the criteria described above, two categories of virtual enterprises can be defined, namely static virtual enterprises and dynamic virtual enterprises (DVEs). In the case of static virtual enterprises the business partners are linked statically, i.e. the relationships between partners are fixed and the shared business processes have pre-defined interfaces, are tightly coupled, and are customised according to the partners' environments and requirements. Changes during the execution of business processes are not foreseen, except for critical situations, like failures or loss of partners.

In dynamic virtual enterprises the business partners are found and linked dynamically, on-demand, and according to the requirements of the customers with the help of the services provided by a virtual market place (Nwana 99). The business relationships in a dynamic virtual enterprise can change continuously based on market-driven criteria. The virtual market place provides services for the registration of business process offerings based on generic, well-known process templates. Business domains that want to engage in virtual enterprise relationships can register offers at the market place in relation to the process templates. Whenever a business domain wants to use a particular process, it is visiting the virtual market place and locates all the potential partners that can provide the required process. As soon as the market place search has resulted in a list of candidate partners, the partner selection process starts. Included in the process are negotiations about the capacities and services required and contracting about the roles and tasks of each partner. The negotiation process can be performed manually, or automatically. The result of it is usually a contract, which regulates the business relationships established. With the support of the virtual market place, the number and role of partners can easily be adapted to the requirements of customers and the currently available capacities and abilities of the partners. This is a significant evolutionary step, which lets virtual enterprises take advantage of the demand and supply regulated by an open, Internetbased market (Schuldt 99).

Consequently, from a business point of view, dynamic virtual enterprises appear to be the most promising approach. However, from the technical point of view, the required solutions and systems are more complex and more sophisticated. Therefore, evaluating and integrating actual information and communication technologies is an important step towards an efficient, flexible, and easy-to-use support environment for dynamic virtual enterprises.

\section{TECHNOLOGIES FOR DYNAMIC VIRTUAL ENTERPRISES}

In the following, we describe technologies of particular interest to dynamic virtual enterprises, including those which support the exchange of information in loosely coupled inter-organisational environments, the co-operation of automated 
business components, the control of work processes across organisational boundaries, and the dynamic mediation of business process providers and users.

\subsection{Information Exchange Between Loosely Coupled Systems}

Systems supporting the information exchange between the loosely coupled systems of dynamic virtual enterprises must enable asynchronous transactions across organisational boundaries, flexibly support application domain-specific types and formats of information, and allow for autonomous behaviour of the communicating and co-operating business processes.

EDI. EDI (Electronic Data Interchange) is a standard format for exchanging business data. An EDI message contains a string of data elements, each of which represents a singular fact, such as a price, or a product model number. One or more of such strings form a transaction set, which is the EDI equivalent to a message. A transaction set often consists of what would usually be contained in a typical business document or form.

EDI transactions are typically performed asynchronously to the execution of business processes. This satisfies one requirement of loosely coupled environments and cross-organisational information exchange in virtual enterprises. The current format of EDI messages is static and cannot be extended easily. The scope and context of EDI documents is limited and rather impossible to change, and thus, it is difficult to use EDI as the basis for general-purpose inter-domain business process execution and management. EDI transactions, as currently defined, only support electronic commerce interactions. The business document definitions provided have been considered inadequate for many other application scenarios. To address this issue, the EDI standards organisations, like EDIFACT and ANSI X.12, have developed sets of documents for various industries and business sectors. Using these document definitions, the customisation required per business relationship can be reduced, though in general, per-relationship integration and customisation work is still required.

Under these circumstances, EDI is best suited for long-term and stable business relationships between organisations, which are powerful enough to make significant investments into electronic relationships. Business processes that are not related to electronic commerce, such as supply chain optimisation or product design, are best performed outside the EDI context. In general, each new EDI relationship requires new customisation and integration work. These relationships are not easily established and return on EDI investment is gained only over long periods of time, not over short-term transactions.

DCBS. Distributed component based systems (DCBSs) are widely used for distributed computing and information processing due to the simplicity, ease of integration and deployment, high degree of distribution, standard underlying 
distributed protocols, like CORBA-IIOP and RMI, and middleware services. Backend systems and clients integrate with the distributed framework using the application programming interfaces (APIs) and object models exposed by the underlying levels of the architecture. While clients are insulated from the APIs of the back-end systems, they are tightly bound to the APIs provided by the framework (Orfali 96).

This design choice has two implications. First, by using object binding as the interaction technique, DCBS applications must be adopted all at once by all participants in the cross-organisation relationship. Upgrades to back-end systems, the component framework, and the business application must be co-ordinated across all participants. Second, because of the tight binding, the same object model must be adopted for communication and co-operation across different business domains. This poses a significant barrier to interworking in cross-organisational environments. Additionally, the DCBS frameworks do not provide a complete solution, but instead serve as the starting point for developers to build applications. By building on the framework, developers can more quickly complete applications and leverage the code in the framework that takes care of many of the mechanical details needed for a successful distributed application. Finally, these choices make the DCBS frameworks most appropriate for deployment inside a single company that needs to link multiple distributed divisions or sites. In general, DCBSs are considered inadequate for use in a dynamic virtual enterprise environments because of their tight coupling model (Tombros 00).

Messaging Systems. Messaging systems, in contrast to DCBSs, are not based on a static and tightly coupled component model and do not require compatible middleware services (Filos 00). Messaging systems separate the interface of services from the corresponding modules, which provide these services and, thus, hide the complexities of the server components and systems from the client. In this way, they support asynchronous and loosely coupled relationships among different business domains.

However, there are some drawbacks with messaging systems, too. One of the key problems is that there exist different proposals for a message specification language, i.e. for specifying the envelope and the content of the message. The protocols currently used actually specify their message envelope in XML and follow their own description approach for the included content. Another problem is the lack of generic messaging standards. This results in incompatibilities among different system implementations and makes the integration of business processes across different domains more difficult. The biggest problem, finally, is the specification of an adequate ontology, i.e. a set of concepts describing entities and interactions, such as things, events, and relations, for an agreed-upon vocabulary for each of the different business sectors (Georgakopoulos 98). Standard ontologies would enable the rapid integration and deployment of messaging systems in different application areas. In general, messaging systems have certain benefits over existing DCBS in 
dynamic virtual enterprises due to the asynchronous and loosely coupled approach, the independence among the interfaces of components and the component bodies, and - if available - the well-defined ontologies.

\subsection{Co-operation of Automated Business Components}

Intelligent mobile agents. Intelligent mobile agents combine many of the benefits offered by messaging systems and DCBSs. Agent systems are loosely coupled and communicate asynchronously (Breugst 98). Messages exchanged are well defined through the FIPA agent communication language standard (FIPA 98). Agents realise the concept of ontologies, which makes them more flexible and autonomous and, agent systems are deployed within a distributed object-oriented framework, like CORBA or Java, and thus can access any type of standard business component.

Through these combined benefits, agent systems support autonomy and flexibility, scalability, and adaptability. Flexibility is supported by the distinct communication and co-operation models which have been developed for agentbased systems, scalability comes from the migration capabilities of agents, and adaptability relies on their inherent intelligence. Furthermore, due to the objectoriented concepts used to implement agent platforms and agents the integration with existing technologies is facilitated (Choy 99).

Intelligent mobile agents can be used in different ways to solve virtual enterprise problems. One way is to use an agent based business process management system which controls and co-ordinates the execution of virtual enterprise business processes in a distributed, autonomous, and flexible way. Another way is to use agents for the dynamic selection of partners and the negotiation phase among different virtual enterprise partners (Bellifernine 99). Agents can also be used to manage and co-ordinate the provision of virtual marketplace services.

Although agent technology is a good candidate for the support of virtual enterprises, there are some problems as well. One of the key issues currently is the requirement for a mobile agent platform for the provision of agent life cycle and migration management services (Martesson 98). Several agent platforms have been developed so far, which, however, are mostly incompatible between each other. Agents sitting on different platforms have difficulties in communication and cooperation. Migration between different platforms is nearly impossible and - if actually achieved - relies on dedicated solutions. Current standardisation activities, like OMG-MASIF and FIPA, deal with these problems. 


\subsection{Control of Work Processes Across Organisational Boundaries}

Workflow Management Systems. Workflow management systems are used to specify, execute, manage, and streamline business processes. Workflow management systems in general provide several functions, which can be used for business process execution and control across the organisational boundaries, existing within a virtual enterprise (Grefen 99). Workflow management systems can monitor and control the execution of hierarchical systems of processes and sub-processes, defined by dedicated business modelling tools and business process specification languages.

However, conventional workflow management systems have certain drawbacks in relation to the virtual enterprise concept. One of the main problems is their limited autonomy and flexibility (Miller 98). So far there are no extensions to the existing business process specification languages towards the support of cross-organisational business processes (Bolcer 99). Furthermore, invocation of remote business processes should be preceded by access control, authorisation, and contract checks. Current workflow systems do not provide such mechanisms. Finally, in current workflow systems, shared business processes are structured inflexibly with respect to sub-processes that have to be executed remotely, i.e. the virtual enterprise partners which are to provide those processes are specified statically. Consequently, this approach is suitable for static virtual enterprises only and not for dynamic ones, where the partners, which are to provide parts of the shared business process are not known in advance, and where the remote domains can be selected dynamically, after negotiation and during the business process execution (Tombros 00).

In dynamic virtual enterprises a workflow management system must deal with the fact that for the same business process specification different instances can exist. For every instance a set of different partners may be selected according to the needs and requirements of the process. Standards, currently proposed, are not directly dealing with market-based cross-organisational business process execution and management (Bolcer 99). Critical open issues, like inter-domain workflow execution and management, business process specification languages for inter-domain business processes, and dynamic selection of workflow providers during process execution are not effectively discussed. A message-based approach with corresponding XML message requests and responses is currently considered as a solution to support the required degree of autonomy and flexibility in dynamic virtual enterprises (Ouzounis 98b).

Agent-based workflow management systems are to solve several of these problems. Control of business processes shared between multiple business domains can be assigned to agents which are either deployed directly in each of the domains involved or can migrate from domain to domain as required by the processes structure. Agents arriving at a domain invoke the required sub-process and after 
successful termination of their task either return back to their home agency or migrate to another domain in order to invoke another sub-process. The remote domains can authenticate and authorise the requesting agents based on electronic contracts that have been established during the negotiation phase. However, most of the issues related to such agent-based workflow management systems, crossorganisational business process execution, and dynamic selection of partners are still under investigation and stable, well-defined solutions and concepts are missing.

\subsection{Dynamic Mediation of Business Process Providers and Users}

Virtual Market Places. Virtual market places are central parts of dynamic virtual enterprises. They increase flexibility and scalability through their search and mediation functions and support the selection of business partners during the establishment and reconfiguration of dynamic virtual enterprises. A market place can administer offerings for business processes made by potential providers and can perform searches for partners satisfying required quality attributes. The search and matchmaking services can be further complemented by advanced services, like electronic negotiation and contracting. With respect to negotiations mechanisms applied to the dynamic and automated selection of business processes, the bidding model and the bargaining model are favoured compared to the auction models.

Intelligent mobile agents can be a profitable technical implementation of such functionality due to their inherent autonomy, adaptability, and learning characteristics (Magedanz 99). However, most current implementations of agentbased virtual market places do not consider emerging FIPA agent standards. Furthermore, most of the negotiation approaches, techniques, and models have basically concentrated on $\mathrm{B} 2 \mathrm{C}$ and $\mathrm{C} 2 \mathrm{C}$ electronic commerce and are not addressing the needs of business-to-business market places and dynamic virtual enterprises. Although some of the above techniques can be extended for the dynamic selection of partners in virtual enterprises based on service templates, this area is considered new and further research is needed. Certain key issues like agent communication language, ontology, negotiation protocol, and negotiation strategy need to be clarified and extended for application in dynamic virtual enterprises.

Despite these problems, intelligent mobile agents can provide the basis for the new generation of open, flexible, autonomous, and distributed systems for the management and execution of business processes in dynamic virtual enterprises. 


\section{DIVE - AGENT-BASED LIFE CYCLE MANAGEMENT FOR DYNAMIC VIRTUAL ENTERPRISES}

In the following, we introduce DIVE, a life cycle management approach for dynamic virtual enterprises. It is to realise dynamic virtual enterprise concepts based on agent technology and virtual market places.

In our case, the virtual enterprise life-cycle model consists of two key phases. These phases are the Business Process Specification and Registration Phase and the Business Process Execution and Management Phase.

\subsection{Business Process Specification and Registration}

During the Business Process Specification Phase a virtual enterprise candidate partner specifies its offer for a business processes. Such a process is a hierarchical structure consisting of the root process and a tree of sub-processes. Sub-processes that cannot be further sub-divided are called tasks. Tasks are the actually activities performed by a resource business object, such as a software component, a device, or a robot. A business process offer can be utilised during the execution and management phase either as a self-contained process or as a sub-process of a defined higher-level process. The specification of business processes is done using the DIVE business process definition language. For every business process, the input parameters, the output parameters, the sub-processes, the tasks, and the conditions among the sub-processes and tasks are specified. Additionally, every sub-process and task is specified as a local or remote entity. Local entities are those, which can be entirely performed by the offering domain, while remote entities are those which must be provided by remote domains. Furthermore, for every specified task the associated business objects are specified.

Within DIVE, each business object is encapsulated and represented by a specific agent, called Resource Provider Agent (RPA). In this way, autonomous agents handle legacy services provided by existing distributed objects that are physically located at different network locations. The result of a business process specification in DIVE is an XML-encoded document, which can be interpreted for execution during the business process execution and management phase. During the process registration phase a business domain which wants to participate in dynamic virtual enterprise relationships registers its processes at the virtual market place. The market place administers the type of the process offer together with the attributes to be taken into account during the process execution and management phase. 


\subsection{Business Process Execution and Management}

After successful registration, the offering business domain becomes a virtual enterprise candidate and the business process can be executed upon request from a customer or from a higher-level business process. When a request arrives, it is directed to a virtual enterprise representative, which creates an instance of the process. First the process description is retrieved from the business process repository. Then it is interpreted and the execution of the process is started. The virtual enterprise representative is realised in DIVE by a Personal User Agent (PUA). Each request is administered by a dedicated PUA. The PUA forwards the request to the DIVE agent-based business process management system, which instantiates, interprets, and executes the business process by means of a set of cooperating agents, called Workflow Provider Agents (WPAs).

Communication and co-operation between the WPA and RPA agents, if they all belong to the same domain, are based upon an intra-domain ontology, which comprises the set of messages defined within the domain for the exchange between WPA and RPA agents.

If a sub-process has been specified as remote, then the corresponding WPA creates a specialised negotiation agent, called Requestor Negotiation Agent (RNA), and sends it to the virtual market place in order to locate potential virtual enterprise candidate partners for the required remote sub-process. Upon request, the virtual market place informs the RNA about all the potential virtual enterprise candidate partners. After that, the RNA starts the negotiation process by contacting the negotiation agents located in each candidate partner. These agents are called Provider Negotiation Agents (PNA). The negotiation process is performed among the RNA and PNAs by using a negotiation protocol and a negotiation ontology. The negotiation protocol used is the FIPA Contract Net protocol, a modified version of the original Contract Net protocol. The result of this negotiation process is the selection and contracting of the best virtual enterprise candidate domain according to the constraint given by both sides and the compromise negotiated.

As soon as a virtual enterprise partner for a particular remote process has been selected and contracted, the PNA returns back to his original location and informs the corresponding WPA agent about the selected partner. Then, the WPA contacts that partner domain and requests the execution of the business process by referring to the contract id that has been fixed during the negotiation process. The virtual enterprise partner domain checks the list of existing contracts and starts the execution of the requested process if a legitimate contract is found. The agent responsible for the access control and authorisation is called Domain Representative (DR) agent.

A customer that has requested the execution of a business process can influence and manage the process during execution time. The main operations that can be performed are suspension, resumption, and termination of the process execution. 
Every request of the customer is served initially by the virtual enterprise representative domain. All the WPA and RPA agents, related to the execution of the business process instance, are suspended, resumed, or terminated according to the customer's request. In addition to the activities within the representative domain, all the remote sub-processes and tasks, which have been started in the context of the business process, are suspended, resumed, or terminated, too. Therefore, similar requests are issued and sent from the virtual enterprise representative to the corresponding virtual enterprise partners. Whenever the DR agent of a virtual enterprise partner gets a request to suspend, resume, or terminate an existing local business process, it checks the contract id, and if correct orders the related internal WPA and RPA agents to resume, suspend, or terminate. In that way, unauthorised requests for process suspension, resumption, or termination are not served. The communication and co-ordination between the different agents responsible for remote business processes are regulated by an inter-domain ontology. The interdomain ontology is actually the set of messages that the agents are allowed to exchange during the execution and management of remote business processes. In addition to the execution control actions provided, the customer can request information about the current status of the business process. Similar to the information flow for execution control, the virtual enterprise representative requests from all agents associated with this process, local or remote, to declare their current status. When a process finally completes its operation, the DR agent of the virtual enterprise representative partner is informed. Then, the DR agent informs the customer by posting to it the output results of the process and other status information, like the time of completion. If a fatal problem occurs during the execution of a process, the WPA agent responsible for the process instance informs the DR agent that the execution of this process cannot be continued and consequently, the WPA agent aborts itself. The DR agent informs either the customer or the associated virtual enterprise partner, about this event and stops the execution of the business process.

\subsection{The DIVE Virtual Marketplace}

The virtual market place plays the role of a third party administrative domain within the virtual enterprise. It provides matchmaking services to the virtual enterprise partners. The virtual market place administers a set of so-called service types, which represent the business process types defined and describe in a consistent way the interface of business processes The market place enables virtual enterprise candidate partners to register and administer offers in relation to defined service types and virtual enterprise representatives to search for potential partners that can provide particular business processes associated with existing service types. For every service type, the name of the type and a set of named properties are specified. The name of the service type is the name of the business process, while 
the input and output parameters of the process are named properties of the service type. Additionally, extra properties, related with the negotiation phase, are included in the service type. Service types are managed by the virtual market place administrator. The service type management includes creation, deletion, modification, and retrieval of service types.

Virtual enterprise candidate partners that want to register their process offerings at the market place must match each offer with an existing service type or must initiate the creation of a new type. For a common understanding between the partners making an offer and the domains looking for matching offers, it is necessary that every service offer is an instance of a given service type. Each offer assigns values to the properties defined by the type. Each candidate domain is responsible for its service offers. Actions, which a candidate domain can perform on its offers, include the registration of an offer, the withdrawal, the listing of offers and the modification of an offer.

Virtual enterprise representatives that want to find suitable partners, which can provide a particular process, retrieve from the market place all the registered offers that satisfy given constraints. The search and matchmaking functions required, are provided by the market place. Therefore, the basic functions, which the market place must provide, include service type management, service offer management, and service offer retrieval management.

\section{CONCLUSIONS}

Based on the development, testing, and validation of the DIVE framework, the following conclusions can be drawn according to the selection of technologies and the design decisions during implementation:

Openness has been achieved due to flexible, XML-based ontologies for the management of shared business processes and the negotiation process. The use of open, interoperable standard technologies like XML, FIPA, FIPA-ACL, and Java also increases the openness of the system.

Dynamicity, flexibility and evolution are due to the dynamic selection of virtual enterprise partners and the automated negotiation during business process execution and management.

Asynchronous and loosely coupled communication has been achieved through communication mechanisms supported by a FIPA platform. In general, the intelligent agents communicate asynchronously and loosely coupled by message exchanges through the FIPA agent communication channel (ACC).

Distribution and scalability are due to the autonomous and distributed execution and management of shared business processes by means of intelligent, autonomous agents located in different administrative domains. 
Autonomy has been achieved due to the asynchronous and loosely coupled communication of agents during the execution and management of business processes.

Intelligence is due to the deployment of artificial intelligence techniques during the business process execution and management. For that reason, special mechanisms have been developed and tested for the integration of rule engines like the JESS rule engine for the assertion of conditions related to the control flow in business processes.

Apart from those benefits, a key drawback has been identified. This drawback is the lack of performance. Performance limitations mostly originate from the extra time required for parsing the messages, the asynchronous message transportation imposed by the FIPA ACC, the migration of agents, and the performance characteristics of the underlying agent platform.

Although the presented work intended to provide a coherent solution for the management of dynamic intelligent virtual enterprises, certain issues are still subject to further improvement and research. These are the negotiation strategy algorithms for automated negotiation and partner selection, fault tolerance and exception handling during the execution of shared business processes, and secure inter-domain communication during inter-domain process execution. These and other open issues are addressed by two international projects, recently initiated, namely COVE (Cove 01) and THINKcreative.

\section{REFERENCES}

Applegate, L. M., C.W. Holsapple, R. Kalakota, F. J. Radermacher, and A. B. Whinston (1996). "Electronic Commerce: Building Blocks of New Business Opportunity". Journal of Organizational Computing and Electronic Commerce, vol. 6, no. 1, Addision Wesley 1994, ISBN 0-201-42289-1.

Bellifernine, F., G. Rimassa, and A. Poggi (1999). "JADE: A FIPA Compliant Agent Framework". 4th International Conference and Exhibition on the Practical Applications of Intelligent Agents and Multi-Agent Systems (PAAM 99), London.

Bolcer, G. A. and G. Kaiser (1999). "SWAP: Leveraging the Web to Manage Workflow". IEEE Internet Computing, Jan/Feb 1999.

Breugst, M., L. Hagen, and T. Magedanz (1998a). "Impacts of Mobile Agent Technology on Mobile Communication System Evolution". IEEE Personal Communications Magazine, vol. 5 , no. 4.

Camarinha-Matos, L. M. and H. Afsarmanesh (eds) (1999). "Infrastructures for Virtual Enterrises. Networking Industrial Enterprises". IFIP TC5 WG5.3 / PRODNET Working Conference for Virtual Enterprises (PRO-VE '99), Porto, Portugal, October 1999, Kluwer Academic Publishers.

Choy, S., M. Breugst, and T. Magedanz (1999). "Beyond Mobile Agents with CORBA Towards Mobile CORBA Objects“, 6th ACTS Conference on Intelligence in Services and Networks (IS\&N), H. Zuidweg et a. (Eds.), IS\&N 99, ISBN: 3-540-65895-5, SpringerVerlag, 1999. 
COVE Project, http://www.uninova.pt/ cove/coveproject.htm

Filos, E. and V. Ouzounis (2000). "Virtual Organisations: Technologies, Trends, Standards and the Contribution of the European RTD Programmes". International Journal of Computer Applications in Technology, Special Issue: "Applications in Industry of Product and Process Modeling Using Standards", Virtual-organisation.net, "Newsletter", Vol. 1, No. 3-4, 1997.

FIPA (1998) http://www.fipa.org/spec/FIPA98.html.

Georgakopoulos, D. (1998). "Collaboration Management Infrastructure for Comprehensive Process and Service Management". International Symposium on Advanced Database Support for Workflow Management, Enschede, The Netherlands, May, 1998.

Gibon, P., J.-F. Clavier, S. Loison (1999). "Support for Electronic Data Interchange in Infrastructures for Virtual Enterprises”. Networking Industrial Enterprises. IFIP TC5 WG5.3 / PRODNET Working Conference for Virtual Enterprises (PRO-VE '99), Porto, Portugal, October 1999, Kluwer Academic Publishers.

Grefen, J., B. Pernini, and G. Sanchez (Eds, 1999). "Database Support for Workflow Management: The WIDE Project". Kluwer Academic Publishers, ISB7923-8414-8.

Lin, F. (1996). "Reengineering the Order Fulfillment Process in Supply Chain Networks: A Multiagent Information Systems Approach" Ph.D. Thesis, University of Illinois at UrbanaChampaign.

Magedanz, T. (Ed.) (1999). Special Issue on "Mobile Agents in Intelligent Networks and Mobile Communication Systems“ in Computer Networks Journal, ELSEVIER Publisher, Netherlands, vol. 31, no. 10, July 1999.

Malone, T. W. and J. F. Rockart (1991). Computers, Networks, and the Corporation. Scientific American vol. 265, no. 3. pp. 128-136. R. Gestner, "Using Objects for Workflow Enabling of Standard Application Software", http://laser.cs.umass.edu/workflow/gestner.html, 1996.

Martesson, N., R. Mackay, and S. Björgvinsson (eds) (1998). "Changing the Ways We Work. Shaping the ICT-Solutions for the Next Century". Conference on Integration in Manufacturing, Göteborg, Sweden, October 1998.

Miller J, A. Sheth, K. Kochut, and D. Palaniswami (1998). "The Future of Web-Based Workflows". International Workshop on Research Directions in Process Technology, Nancy, France.

Nwana H, D. Ndumu, L. Lee, and J. Collis (1999). "ZEUS: A Toolkit for Building Distributed Multi-Agent Systems". In: Applied Artifical Intelligence Journal, vol 13, no. 1, 1999. http://www.labs.bt.com/projects/agents/index.htm.

Ouzounis, V. (1998a). "Electronic Commerce Commercial Scenarios, Business Models and Technologies for SME's", European Multimedia, Microprocessor Systems and Electronic Commerce Conference and Exposition (EMMSEC 98), Bordeaux, France September 1998.

Ouzounis, V. (1998b). "Electronic Commerce and New Ways of Work - An R\&D

RoadMap", European Commission -Directoral General III, 1998.

Ouzounis, V. and V. Tschammer (1999). "A Framework for Virtual Enterprise Support Services". 32nd International Conference on System Sciences (HICSS-32), Maoui, Hawaii, January 1999.

Orfali, R. (1996). “The Essential Distributed Objects Survival Guide”, John Wiley and Sons, ISBN 0-471-12993-3, 1996.

Schuldt, H., H. J. Schek, and G. Alonso (1999). "Transactional Coordination Agents for Composite Systems." International Database Engineering and Applications Symposium (IDEAS'99). Montreal, Canada, August 1999. 
Stricker, C., S. Riboni, M. Kradolfer, and J. Taylor (2000). "Market-based Workflow Management for Supply Chains of Services". 33rd International Conference on System Sciences (HICSS-33), Maui, Hawaii, January 2000.

Tombros, D. and A. Geppert (2000). "Building Extensible Workflow Systems Using an Event-Based Infrastructure". 12th Conference on Advanced Information Systems Engineering, Stockholm, Sweden, June 2000. 